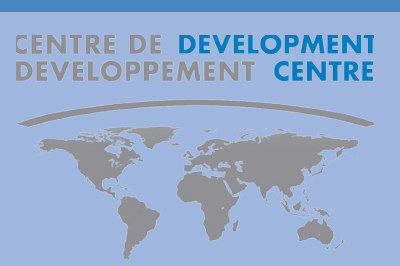

\title{
Repéres
}

$n^{\circ} 65$

avril 2008

\section{Lutter contre la mortalité des enfants de moins de cinq ans en Afrique}

\section{(Rédigé à partir du rapport Perspectives économiques africaines 2008*)}

par Audrey Verdier-Chouchane

- Il est urgent de réduire de deux tiers le taux de mortalité des enfants de moins de cinq ans en Afrique.

- Le VIH-SIDA, la malaria, le manque de services de santé de base et les conflits ralentissent les progrès.

- Des maladies que l'on peut prévenir sont responsables d'une grande partie des décès chez les moins de cinq ans.

- La prévention de la mortalité infantile est une question de volonté politique.

Il y a eu des progrès vers les Objectifs du millénaire pour le développement (OMD) - y compris en direction de l'objectif numéro 4 visant à "réduire de deux tiers la mortalité des enfants de moins de cinq ans" - lorsque les gouvernements ont fait preuve de fermeté dans la direction et lorsque des politiques adaptées ont par ailleurs été accompagnées de soutiens financiers et techniques adéquats.

Cela peut être fait.

William Easterly avait raison en $2007^{1}$ lorsqu'il affirmait qu'une interprétation unidimensionnelle des objectifs du millénaire dessinait un portrait exagérément pâle de I'Afrique. Il considère que les OMD ont été mal conçus et qu'ils laissent une place à l'arbitraire, ce qui fait que les performances relatives des pays africains paraissent particulièrement faibles. Les taux de mortalité infantile en Afrique étant déjà très élevés lorsque les objectifs ont été établis, il n'était que peu probable qu'une réduction de deux tiers soit faisable. Le taux de mortalité des moins de cinq ans atteignait en moyenne $154 \%$ en 1990 en Afrique, ce qui voudrait dire qu'il faille le réduire à $51 \%$ o d'ici 2015 pour atteindre l'objectif fixé. En 2005, sur le continent, le taux de mortalité des enfants de moins de cinq ans a atteint $137 \%$, ce qui représente une baisse de 9.3 pour cent au lieu des 40 pour cent nécessaires pour suivre le rythme demandé.

Les principales raisons de ce retard et de la lenteur des progrès dans le domaine sont le VIH/SIDA, la malaria, le manque de services de santé de base et la permanence de situations conflictuelles. Dans les pays du sud de l'Afrique, où la pandémie du SIDA est élevée, les taux de mortalité ont été plus élevés en 2005 que dans les années 1990. (Figure 1).

1. Easterly, William (2007) "How the Millennium Development Goals are unfair to Africa", Global Economy and Development, Working Paper 14, novembre 2007.
Figure 1. Taux de mortalité des enfants de moins de cinq ans dans certains pays du sud de l'Afrique

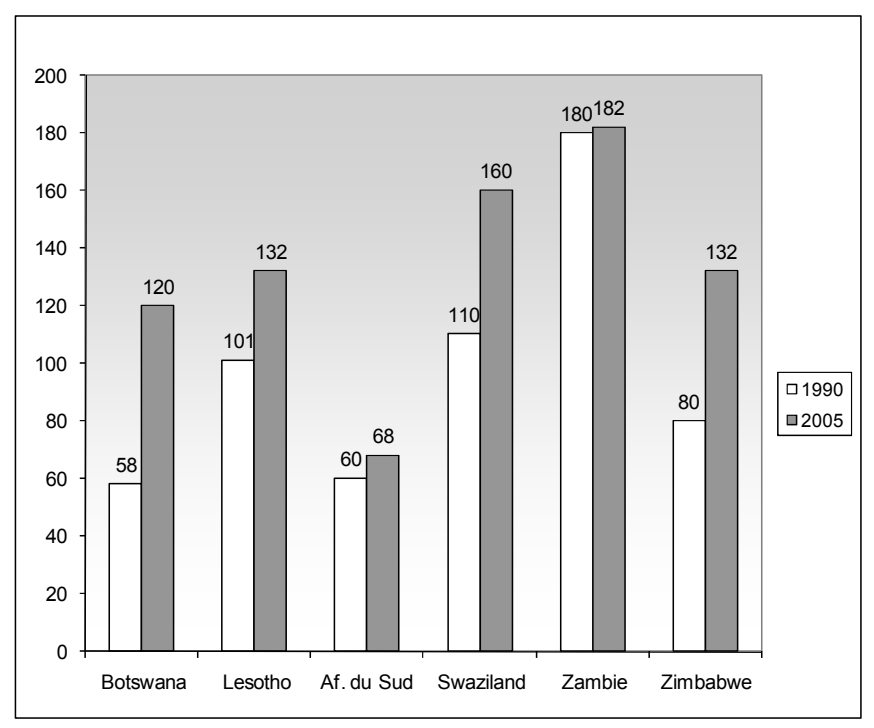

Source: Graphique réalisé par l'auteur à partir de données de la Banque mondiale disponibles en ligne, World Development Indicators, Washington, D.C.

Ces chiffres sont très mauvais quels que soient les standards et ils nécessitent que des mesures urgentes soient prises. Certains pays ont répondu et ont adopté des politiques adéquates qui commencent à faire leurs preuves, même si cela ne les remet pas encore vraiment sur les rails. Ils sont cependant la preuve que des progrès substantiels sont possibles.

En Afrique, plus généralement, seuls 17 pour cent des pays ont atteint les objectifs ou sont sur la bonne voie pour le faire. Lorsque I'on retire les pays d'Afrique du nord les plus performants, le taux passe à 7.5 pour cent. 
L'Égypte a déjà atteint l'objectif, et I'Algérie, le Cap-Vert, les Comores, l'Érythrée, la Libye, le Malawi, le Maroc et la Tunisie vont certainement y parvenir (Figure 2). Le taux de mortalité infantile diminue, sans surprise, dans les foyers les plus riches, dans les zones urbaines, et au sein des foyers dans lesquels la mère est éduquée.

Le vaccin contre la rougeole est un autre facteur important de la baisse du taux de mortalité infantile. En 2005, dans toute l'Afrique, seuls 64 pour cent des enfants entre 12 et 23 mois avaient reçu au moins une dose de vaccin contre la rougeole (contre 95 pour cent en Afrique du Nord). Le continent peut faire mieux que cela.

Figure 2. Meilleures performances face à l'objectif $n^{\circ} 4$ (Évolution des taux de mortalité chez les moins de cinq ans)

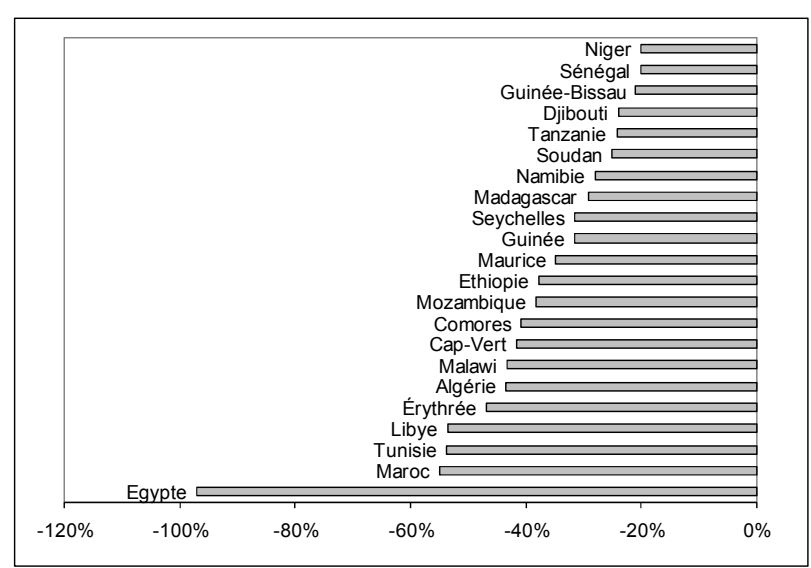

Source: Graphique réalisé par l'auteur à partir de données de la Banque mondiale disponibles en ligne, World Development Indicators, Washington, D.C.

Quels sont donc les pays qui avancent vers I'OMD 4 et comment font-ils pour y parvenir?

Selon I'ONG Save the Children², ces pays ont du mérite à avoir sauvé des vies d'enfants de moins de cinq ans. En dépit de leur PIB per capita très faible, Madagascar, le Malawi et la Tanzanie ont enregistré une baisse respective de 32,46 et 27 pour cent du taux de mortalité des enfants de moins de cinq ans entre 1990 et 2006. Le rôle des gouvernements y est déterminant. En effet, cette baisse a été réalisée grâce à des réformes du secteur de la santé et à des programmes communautaires. Au Malawi et en Tanzanie, le progrès est partiellement le résultat d'une amélioration des compétences des travailleurs du secteur sanitaire et à celle des soins pendant la grossesse. À cela s'est ajouté le fait que les enfants de 5 à 59 mois ont reçu au moins un supplément de vitamine $A$ pour lutter contre la malnutrition. À Madagascar, la stratégie principale a été de prévenir la malnutrition en encourageant l'allaitement.

Le cas égyptien démontre encore mieux comment la volonté politique est la clef du succès. L'Égypte est en effet le seul pays africain à avoir atteint, dès 2006, l'objectif n 4. Dès 1992, le gouvernement a lancé une initiative majeure appelée "mère en bonne santé, enfant en bonne santé" afin d'améliorer les conditions sanitaires dans les zones les plus pauvres. Les soins pendant la grossesse ont été fortement améliorés, l'accueil et l'assistance lors des accouchements se sont professionnalisés, et l'accès à l'information et aux services de planning familial s'est répandu.

Ces exemples prouvent que la réduction des taux de mortalité des enfants est à la portée des pays africains. Cinq mesures simples, recommandées entre autres par Save the Children peuvent servir : former les médecins et les infirmières aux accouchements, encourager l'allaitement pendant les six premiers mois de la vie du nourrisson, généraliser le vaccin contre la rougeole, encourager la réhydratation orale pour lutter contre la diarrhée, et former les travailleurs sanitaires à diagnostiquer les pneumonies et à les soigner avec des antibiotiques.

Tout ceci peut être fait.

2. Save the Children (2007) State of the World's Mothers Index: Save the Lives of Children under 5, disponible sur : www.savethechildren.org

* Il s'agit d'un rapport conjoint de la Banque africaine de développementet du Centre de développement de I'OCDE, avec la Commission Économique pour l'Afrique des Nations unies, qui a bénéficié du soutien financier de la Commission européenne.

Les lecteurs sont invités à citer ou reproduire les informations des Repères du Centre de développement de I'OCDE dans leurs propres publications. En échange, le Centre demande les remerciements de rigueur ainsi qu'un exemplaire de la publication. Le texte intégral des Repères et d'autres informations sur le Centre de développement et ses travaux sont disponibles sur : www.oecd.org/dev
Centre de développement de I'OCDE 2, rue André-Pascal, 75775 Paris Cedex 16, France Tél : $33(0) 145.24 .82 .00$

Fax : $33(0) 144.30 .61 .49$ mél : cendev.contact@oecd.org 